\title{
Análise da Revisão Cochrane: Uso de Corticoterapia Sistémica nas Exacerbações Agudas da Doença Pulmonar Obstrutiva Crónica. Cochrane Database Syst Rev. 2014, 9:CD001288
}

\author{
Analysis of the Cochrane Review: Use of Systemic Corticosteroids for Acute \\ Exacerbations of Chronic Obstructive Pulmonary Disease. Cochrane Database \\ Syst Rev. 2014, 9:CD001288
}

\author{
Pedro AZEVEDO ${ }^{1}$, João COSTA ${ }^{1,2,3}$, António VAZ-CARNEIRO1,2 \\ Acta Med Port 2014 Sep-Oct;27(5):537-540
}

\section{RESUMO}

As exacerbações agudas da doença pulmonar obstrutiva crónica são uma das principais causas de internamentos hospitalares e de mortalidade, contribuindo para o declínio da função pulmonar, capacidade física e qualidade de vida. As infecções são a causa principal das exacerbações e o tratamento inclui antibióticos, broncodilatadores e corticóides sistémicos como agentes anti-inflamatórios. A presente revisão da Cochrane compara: 1 . O uso de corticoterapia oral e parentérica com o uso de placebo; 2 . As vias de administração entre si. Os resultados indicam que existe evidência a favor do uso de corticoterapia no tratamento das exacerbações da doença pulmonar obstrutiva crónica já que melhora precocemente a função pulmonar [avaliada pelo volume expiratório forçado no primeiro segundo $\left(F E V_{1}\right)$ ], reduz a probabilidade de falência terapêutica e de recidiva no primeiro mês, encurta a hospitalização em doente que não requerem regime de cuidados intensivos. Contudo, a corticoterapia acarreta um aumento de eventos adversos associados ao fármaco, nomeadamente hiperglicémias, sobretudo se a via de administração for parentérica. A via parentérica não mostrou ser superior à via oral na falência terapêutica, recidiva ou mortalidade. A mortalidade até aos 30 dias não parece ser afectada pelo uso de corticóides.

Palavras-chave: Corticosteróides; Doença Pulmonar Obstrutiva Crónica/ tratamento; Doença Pulmonar Obstrutiva Crónica/complicações; Ensaios Clínicos Controlados Aleatórios como Assunto; Revisão Sistemática.

\section{ABSTRACT}

Acute exacerbations of chronic obstructive pulmonary disease are a major cause of hospital admissions and mortality, contributing to the decline in lung function, exercise capacity and quality of life. Infections are the major cause of exacerbations and treatment includes antibiotics, bronchodilators and systemic corticosteroids as anti-inflammatory agents. This Cochrane review compared: 1. Use of oral and parenteral corticosteroids with placebo use; 2. Routes of administration among themselves. The results indicate that there is evidence for the use of corticosteroids in the treatment of chronic obstructive pulmonary disease exacerbations since early improvement in lung function [assessed by forced expiratory volume in one second $\left(F E V_{1}\right)$ ] has been noted, the likelihood of treatment failure and relapse in the first month has been reduced and it shortens the hospital stay in patients who do not require intensive care regimen. However, corticosteroid therapy causes an increase in adverse effects associated with drug, namely hyperglycaemia, especially if the route of administration is parenteral. Parenteral route has not shown to be superior to oral route in treatment failure, relapse, or death. Mortality up to 30 days does not seem to be affected by the use of corticosteroids.

Keywords: Adrenal Cortex Hormones; Pulmonary Disease, Chronic Obstructive /drug therapy; Pulmonary Disease, Chronic Obstructive/complications; Randomized Controlled Trials as Topic.

\section{QUESTÃO CLÍNICA}

O uso de corticóides sistémicos reduz as exacerbações agudas da DPOC?

\section{OBJECTIVOS}

Esta revisão sistemática procurou avaliar os potenciais efeitos benéficos (eficácia) e prejudiciais (eventos adversos) da corticoterapia oral e parentérica versus placebo no tratamento das exacerbações agudas da doença pulmonar obstrutiva crónica (DPOC), comparando ainda a eficácia das duas vias de administração.

\section{CRITÉRIOS DE SELECÇÃo}

Foram seleccionados ensaios clínicos controlados e aleatorizados (RCTs) que compararam a administração oral e parentérica com o uso de placebo, ou que compararam corticoterapia oral e parentérica no tratamento de pessoas com exacerbação aguda da DPOC. Em ambos os grupos as outras terapêuticas (p.e. broncodilatadores e antibióticos) foram padronizadas. Excluíram-se ensaios clínicos sobre asma agudizada.

\footnotetext{
1. Centro de Estudos de Medicina Baseada na Evidência. Faculdade de Medicina. Universidade de Lisboa. Lisboa. Portugal.

2. Centro Colaborador Português da Rede Cochrane Iberoamericana. Lisboa. Portugal.

3. Unidade de Farmacologia Clínica. Instituto de Medicina Molecular. Faculdade de Medicina. Universidade de Lisboa. Lisboa. Portugal.

Recebido: 02 de Outubro de 2014 - Aceite: 02 de Outubro de 2014 | Copyright @ Ordem dos Médicos 2014
} 


\section{METODOLOGIA}

Foram realizadas pesquisas em várias bases de dados: Cochrane Airways Group Specialised Register of Trials, Medline e Central (Cochrane Central Register of Controlled Trials). Verificaram-se as referências dos estudos incluídos e os registos dos ensaios. A última pesquisa foi efectuada em Maio de 2014.

\section{RESULTADOS}

Incluíram-se nesta revisão sistemática 16 estudos ( $n=1787$ doentes) que compararam o uso de corticoterapia sistémica com o uso de placebo [13 estudos $(n=1620)$ contribuíram com resultados para a meta-análise] e quatro estudos $(n=298)$ que compararam o uso de corticoterapia por via oral com a via endovenosa [três estudos $(n=239)$ contribuíram com resultados para a metaanálise]. A idade média dos participantes foi de 68 anos (82\% do sexo masculino) e o $\mathrm{FEV}_{1}$ médio na admissão foi de $40 \%$ [seis estudos $(n=633)$ ]. A maioria dos estudos decorreu em ambiente hospitalar, dois decorreram em unidades de cuidados intensivos e três foram realizados em doentes tratados no domicílio. O viés de selecção foi considerado negligenciável em todos os estudos. Foi considerado existir alto viés de desempenho em um estudo que comparou corticoterapia sistémica com placebo e em dois estudos que compararam corticoterapia por via oral com a via endovenosa.

Em nove estudos ( $n=917$ ) houve evidência de alta qualidade para a associação entre o uso de corticoterapia sistémicas e a redução do risco de falência terapêutica em cerca de $50 \%$ versus placebo (média de 14 dias de terapêutica; odds ratio [OR] 0,48; intervalo de confiança [IC] 95\%: 0,35-0,67). Foi considerado necessário tratar nove pessoas (IC 95\%: 7-14) com corticoterapia sistémica para evitar uma falência terapêutica. Em dois estudos ( $n=$ 415) houve evidência moderada da menor taxa de recidiva com um mês de terapêutica com corticoterapia sistémica [hazard ratio (HR) 0,75; IC 95\%: 0,63-0,95]. Em 12 estudos $(n=1319)$ a corticoterapia sistémica não mostrou ser superior ao placebo na redução da mortalidade até aos 30 dias [OR 1,00; IC 95\%: 0,6-1,66].

A corticoterapia mostrou melhoria no $\mathrm{FEV}_{1}$ nos primeiros três dias em sete estudos $(n=646$; diferença média 140 cc; IC 95\%: 90-200), o que não se verificou para lá desse período. Verificou-se ainda melhoria nos sintomas de dispneia e nos gases do sangue $\left(\mathrm{PaO}_{2} \mathrm{e}\right.$ $\mathrm{PaCO}_{2}$ ) nos primeiros três dias.

O risco de eventos adversos foi cerca do dobro com a corticoterapia (OR 2,33: IC 95\%: 1,59-3,43), existindo uma relação de um efeito adverso para cada seis doentes tratados (IC 95\%: 4-10), com especial destaque para o risco de hiperglicémia (OR 2,79; IC 95\%: 1,86-4,19). Contudo, quando comparado com o grupo controlo, a duração da hospitalização foi mais curta 1 a 2 dias nos doentes tratados com corticóides [média 1,22 dias; IC 95\%: 2,26- $(-0,18)$ ]; esta redução não se verificou nos doentes que necessitaram de ventilação assistida.
Não se verificaram diferenças nos outcomes primários de eficácia (falência terapêutica, recidiva e mortalidade) nem nos outcomes secundários, comparando os doentes sob corticoterapia oral e os doentes sob corticoterapia parentérica. Um estudo mostrou aumento significativo do risco de hiperglicémia nos doentes tratados com corticóides por via parentérica (OR 4,89; IC 95\%: 1,2019,94).

$\mathrm{Na}$ Tabela 1 estão descritos os resultados para a comparação principal.

\section{CONCLUSÕES}

Actualmente existe evidência de que o uso de corticóides por via oral ou por via parentérica nas exacerbações aguda da DPOC reduz o risco de falência terapêutica e de recidiva no primeiro mês, encurta a hospitalização em doentes que não requerem regime de cuidados intensivos e melhora precocemente a função pulmonar e os sintomas associados à doença. A via parentérica não mostrou ser superior à via oral na falência terapêutica, recidiva ou mortalidade. Existe um aumento do risco de eventos adversos associados ao fármaco em doentes sob corticoterapia, especialmente naqueles sob via endovenosa.

\section{COMENTÁRIO}

A DPOC tem morbilidade e mortalidade crescentes, prevendo-se que em 2020 seja a terceira principal causa de morte a nível mundial. ${ }^{1} \mathrm{O}$ bom controlo desta patologia passa, sobretudo, pela prevenção da doença investindo na evicção tabágica. ${ }^{2}$ Uma vez estabelecida a doença, a mitigação das exacerbações por via de um bom controlo farmacológico deve ser a principal preocupação.

A presente revisão sistemática teve por base estudos que compararam o uso de corticoterapia por via oral e por via endovenosa entre si e com o uso de placebo no controlo das exacerbações.

Os resultados globais foram claramente favoráveis ao uso de corticóides uma vez que melhora a função pulmonar, reduz as recidivas e o tempo de internamento. As diferenças encontradas são clinicamente relevantes. Apesar da corticoterapia estar associada a um aumento do risco de alguns eventos adversos, como por exemplo retenção de fluidos, hipertensão, diabetes mellitus, supressão adrenérgica, osteoporose e aumento do risco de fracturas, este risco deve ser equilibrado com benefícios documentados nesta revisão sistemática.

São necessários mais estudos sobre a frequência das exacerbações e o uso repetitivo de corticoterapia e o tratamento dos doentes em ambiente de cuidados intensivos (que necessitam que ventilação assistida)

\section{IMPLICAÇÕES PARA A PRÁTICA}

1. O uso de corticoterapia parece ter impacto clinicamente significativo no tratamento das exacerbações agudas da DPOC melhorando precocemente a função pulmonar, reduzindo as recidivas e diminuindo a duração 
Tabela 1 - Resumo dos resultados para a comparação principal

Corticoterapia sistémica versus placebo nas EA da DPO
Doente ou população: exacerbações agudas da DPOC
Situação: ambulatório, enfermaria e cuidados intensivos
Estudo: corticoterapia sistémica
Controlo: placebo

\begin{tabular}{|c|c|c|c|c|c|c|}
\hline \multirow{3}{*}{ Outcomes } & \multicolumn{2}{|c|}{ Riscos comparativos ilustrativos* (IC 95\%) } & \multirow[b]{2}{*}{$\begin{array}{l}\text { Efeito relativo } \\
\text { (IC 95\%) }\end{array}$} & \multirow[b]{2}{*}{$\begin{array}{c}\mathrm{N} .^{\circ} \text { de } \\
\text { participantes } \\
\text { (estudos) }\end{array}$} & \multirow[b]{2}{*}{$\begin{array}{c}\text { Qualidade } \\
\text { da evidência } \\
\text { (GRADE) }\end{array}$} & \multirow[b]{2}{*}{ Comentários } \\
\hline & $\begin{array}{l}\text { Risco } \\
\text { assumido }\end{array}$ & $\begin{array}{c}\text { Risco } \\
\text { correspondente }\end{array}$ & & & & \\
\hline & & $\begin{array}{l}\text { Corticoterapia } \\
\text { sistémica }\end{array}$ & & & & \\
\hline
\end{tabular}

\section{Falência}

terapêutica

Necessidade de intensificar a terapêutica / SU ou

276 por 1000

154 por 1000
$(118$ a 203)

OR 0,48
$(0,35$ a 0,67$)$

917 (9 estudos)

++++
alta

Follow-up: 3-30 dias

Recaída

Tratamento para

exacerbações agudas da

DPOC ou re-admissão

hospitalar

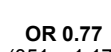

OR 0.77
$(051$ a 1,17$)$

595

(5 estudos)

$\stackrel{+++}{\text { moderada }^{1}}$

Em 2 estudos

$(n=415)$ a recaída ao $1^{\circ}$

mês foi mais reduzida com

corticoterapia sistémica

comparando som o placebo (HR 0,78; IC 95\% 0,63-0,97)
Follow-up: 1-4 meses

\section{Melhoria na função} pulmonar - efeito precoce

$\mathrm{FEV}_{1}(\mathrm{~L})$ absoluto ou variação

Follow-up: 3 dias
$\mathrm{FEV}_{1}$ médio no grupo-controlo variou de 0,77 $0,91 \mathrm{~L}$
A melhoria da função pulmonar média no grupo-estudo foi
$\mathbf{0 , 1 4} \mathrm{L}$ superior 0,14 L superior
$(0,09$ a 0,20 maior $)$

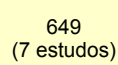

$++++$ (7 estudos) $\begin{array}{ll}\text { A variação média } & \text { na sensação }\end{array}$ de dispneia no de dispneia no A variação média na dispneia - efeito precoce de 1,8 unidades na escala de redução da sensação Escala de Borg ou VAS na escala estudo foi 0,35 desvios-padrão unidades na $\quad$ superior Follow-up: 3 dias unidades na

481 por 1000
$(388$ a 577$)$

282 por 1000
$(208$ a 371$)$
OR 2,33
$(1,59$ a 3,43$)$

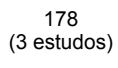
$\begin{array}{ccc} & 178 & +++ \\ - & \text { (3 estudos) } & \text { moderada }^{2}\end{array}$

Efeito da amostra na escala de Borg 0,93 unidades; IC 95\% 0,18-1,7 (DMCI = 2); efeito da $95 \% 0,75-9,59(\mathrm{DMCl}=10)$
Efeito adverso do fármaco

Follow-up: 2-26 semanas

Hiperglicémia
285 por 1000

124 por 1000
OR 2,79
$(1,86$ a 4,19
736 (8 estudos)

++++
alta

*A base para o risco assumido (por exemplo, o risco grupo controle mediana entre os estudos) é fornecido em notas de rodapé. O risco correspondente (e o seu intervalo de confiança de $95 \%$ ) é baseado no risco assumido no grupo-controlo e o efeito relativo da intervenção (e o seu IC de $95 \%$ ).

EA: exacerbação aguda; IC: intervalo de confiança; DPOC: doença pulmonar obstrutiva crónica; SU: serviço de urgencia; FEV $_{1}$ : volume expiratório forçado no $1^{\circ}$ segundo; HR: hazard ratio (taxa de risco); DMCI: diferença mínima clinicamente importante; OR: odds ratio; VAS: escala visual analógica.

Grupo de Trabalho GRADE - níveis de evidência

Alta qualidade: é muito improvável que estudos futuros mudem a confiança na estimativa de efeito.

Moderada qualidade: mais pesquisas são susceptíveis de ter um impacto importante sobre a confiança na estimativa do efeito e pode mudar a estimativa.

Baixa qualidade: mais pesquisas são muito propensas a ter um impacto importante sobre a nossa confiança na estimativa do efeito e provavelmente mudará a estimativa.

Muito baixa qualidade: existem muitas incertezas sobre a estimativa.

IC alargados incluem benefícios significativos e danos (-1 para imprecisão)

$\mathrm{Cl}$ superior ou inferior do tamanho do efeito cruza 0,5 (-1 para imprecisão).

da hospitalização (fora do ambiente de cuidados intensivos).

2. Não existe diferença em termos de eficácia no uso de corticoterapia oral e parentérica.

3. A corticoterapia, especialmente parentérica, está associada a eventos adversos relacionados com o fármaco, nomeadamente hiperglicémia. Contudo, estes efeitos não parecem persistir depois de finalizado o tratamento. 


\section{REFERÊNCIAS}

1. Lokke A, Lange P, Scharling H, Fabricius $P$, Vestbo J. Developing COPD: a 25 year follow up study of the general population. Thorax 2006;61:935-9.

2. Global strategy for the diagnosis, management, and prevention of chronic obstructive pulmonary disease: Revised 2014. Global Initiative for Chronic Obstructive Lung Disease (GOLD). [Consultado 2014 Set 30]. Disponivel em:http://www.goldcopd.org.

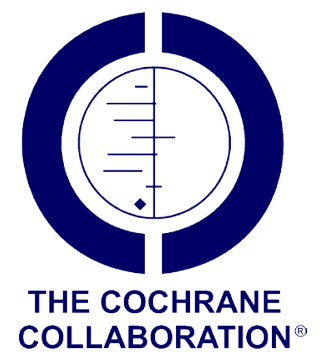




\section{Análise da Revisão Cochrane: Uso de Corticoterapia Sistémica nas Exacerbações Agudas da Doença Pulmonar Obstrutiva Crónica. Cochrane Database Syst Rev. 2014, 9:CD001288}

Acta Med Port 2014:27:537-540

Publicado pela Acta Médica Portuguesa, a Revista Científica da Ordem dos Médicos

Av. Almirante Gago Coutinho, 151

1749-084 Lisboa, Portugal.

Tel: +351218428215

E-mail: submissao@actamedicaportuguesa.com

www.actamedicaportuguesa.com

ISSN:0870-399X | e-ISSN: 1646-0758

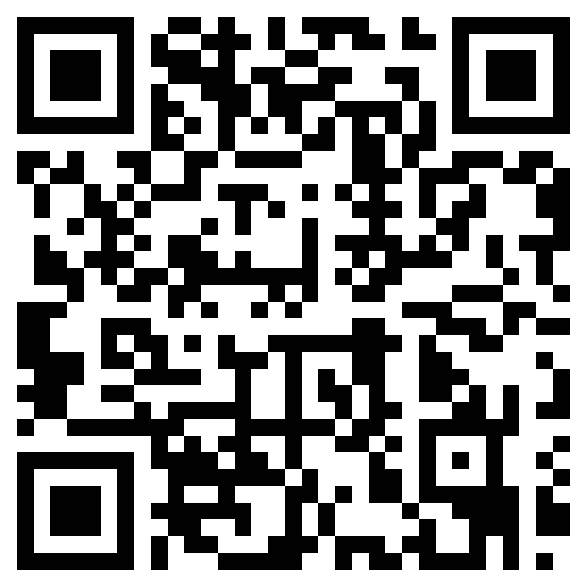

